\title{
Spectrum of Electrocardiographic Changes in Patients Presenting with Acute Organophosphate Compounds Poisoning
}

\author{
Sonali Nayak ${ }^{1}$, Roshan Kurmi ${ }^{2}$ \\ ${ }^{1}$ Department of Internal Medicine, B.P. Koirala Institute of Health Sciences (BPKIHS), Dharan, Sunsari, Nepal. \\ ${ }^{2}$ Department of Internal Medicine, National Medical College and Teaching Hospital, Birgunj, Parsa, Nepal.
}

\section{ABSTRACT}

\section{BACKGROUND}

Organophosphate compounds (OPC) are important cause of poisoning in developing countries. They are irreversible cholinesterase inhibitors and produce signs and symptoms pertaining to various organ systems via their muscarinic and nicotinic effects. The cardiac manifestations include hemodynamic instability and various arrhythmias. Here, in this study, we described various electrocardiographic (ECG) changes in patients of OPC poisoning in our population.

\section{METHODS}

This is a descriptive, cross-sectional study conducted at tertiary academic centre. All patients ( $>14$ years and $<75$ years) with history of OPC poisoning were included. Diagnosis was made on the basis of history and clinical features. ECG analysis of each patient was done for rate, rhythm, ST segment and T wave changes, PR and QTc interval, conduction defects and atrial and ventricular premature complexes before and after administration of atropine.

\section{RESULTS}

A total of 54 patients with OPC poisoning were included. The mean age was $28 \pm$ 14.5 years. The predominant age group was $<30$ years. There was female predominance with male : female ratio of $1: 1.45$. The most common ECG finding was sinus arrhythmia (tachycardia $25.9 \%$ and bradycardia $20.4 \%$ ), followed by prolonged QTc (14.8\%), ST - T changes (11.1\%), premature ventricular complexes (PVCs) (7.4\%), prolonged PR interval (3.7\%) and atrial fibrillation (1.9\%). There was no mortality. The mean ICU and Hospital stay was three and six days respectively.

\section{CONCLUSIONS}

Sinus arrhythmia was the most common ECG changes followed by prolonged QTc, ST - T segment changes and PVCs in our setup. Careful observation of these ECG changes and timely intervention can prevent from sudden cardiac death in these patients.

\section{KEY WORDS}

Cardiac, Complications, Electrocardiogram, Organophosphates, Poisoning
Corresponding Author:

Dr. Sonali Nayak,

\#92-B; B.P. Koirala Institute of Health

Sciences (BPKIHS), Dharan

Sunsari, Nepal.

E-mail: sonalinayak330@gmail.com

DOI: $10.14260 /$ jemds/2021/708

How to Cite This Article:

Nayak $N$, Kurmi $R$. Spectrum of electrocardiographic changes in patients presenting with acute organophosphate compounds poisoning. J Evolution Med Dent Sci 2021;10(39):3496-3500, DOI: 10.14260/jemds/2021/708

Submission 27-06-2021,

Peer Review 11-09-2021,

Acceptance 19-09-2021,

Published 27-09-2021.

Copyright (C) 2021 Sonali Nayak et al. This is an open access article distributed under Creative Commons Attribution License [Attribution 4.0 International (CC BY 4.0)] 


\section{BACKGROUND}

Organophosphorus compounds (OPC), widely - used as insecticides and pesticides, are common causes of poisoning in agrarian countries including Nepal.1,2 Nearly half of the acute poisoning are due to OPC in various hospital based studies. ${ }^{3}$ In developing countries where the medical facilities are limited, fatality rate remains higher. It is an important preventable public health problem in these regions. Although accidental poisoning can occur following exposure or inhalation, serious poisoning often follows suicidal ingestion due to its easy accessibility, which is often preferred by young economically productive age group with a high case fatality rate. 4,5 As per World Health Organization (WHO) estimates, about 3 million people are being exposed to pesticide poisoning every year, with about 2,00,000 deaths per year in developing countries. Nearly $90 \%$ of the poisoning are suicidal with a fatality rate of $>10 \% ; 8-10 \%$ are accidental and $<1 \%$ are homicidal. Occupational exposure accounts for nearly $20 \%$ of accidental poisoning with fatality rate of $<1$ $\% .2,5$

OPCs constitute a heterogeneous category of chemicals designed for the control of pests, weeds and plant diseases. They are irreversible inhibitor of acetylcholine esterase (AChE) and cause excessive cholinergic stimulation via the muscarinic and nicotinic receptors found in the central and peripheral nervous system and neuro-muscular junctions of skeletal muscle. The commonly used OPCs are chlorpyriphos, cypermethrin, dichlorovos, methylparathiion, dimethoate, malathion, monocrotophos and fenthion. OPCs can easily cross the respiratory epithelium, dermal and gastric mucosa because of their lipophilic structure. The manifestations of OPC poisoning depend on type (muscarinic or nicotinic) and site (central or peripheral) of receptors involved. Different organ systems are affected in various degrees, predominant organ system involved being respiratory, nervous and cardiovascular system.6,7,8 The muscarinic symptoms and signs are more frequent ( $84 \%$ ) followed by central nervous system (78 \%) and nicotinic (17\%). Clinical effects of OPCs poisoning occur in three phases. The first is acute cholinergic crisis phase, second is delayed complications like polyneuropathy and a phase in between these two is known as intermediate syndrome. ${ }^{8}$

Cardiac manifestations are observed in about two-third of patients with OPC poisoning, which can be serious and fatal. These complications are potentially preventable, if recognized early and treated adequately. Common electrocardiographic findings are QTc prolongation, ST - T segment changes, various degrees of heart block, and atrial fibrillation. It can cause fatal arrhythmias like ventricular premature complexes (VPCs) and ventricular tachycardia and fibrillation. Other cardiac manifestations include hypotension, bradycardia, tachycardia, hypertension and non-cardiogenic pulmonary oedema. ${ }^{9,10}$ Electrocardiographic changes in OPC poisoning have been reported along with the associated structural myocardial damage. The mechanism of cardiac toxicity is multifactorial. Hypoxemia, acidosis and electrolyte derangements, sympathetic and parasympathetic overactivity, direct toxicity on myocardium and conduction system of the heart are common predisposing factors. OPC poisoning itself causes diarrhoea and vomiting which can lead to electrolyte derangements that may cause electrocardiographic changes. ${ }^{11,12}$

This study is undertaken to determine the spectrum of the electrocardiographic changes in patients presenting with OPC poisoning.

\section{METHODS}

This is a descriptive, cross-sectional study conducted at National Medical College and Teaching Hospital (NMCTH), Birgunj, Parsa, Nepal over a period of 12 months from November 2016 to October 2017. Ethical approval was obtained from the Institutional Review Committee (IRC) of NMCTH prior to the start of the study. Written informed consent was obtained from patients or their relatives prior to enrolment in the study.

The objective of the study was to determine the spectrum of electrocardiographic changes in patients of OPC poisoning. Non-probability purposive sampling was done and sample size was calculated to be 54 by using Solven's formula.

All patients with clinical evidence of acute organophosphate poisoning presenting to the emergency department of NMCTH during data collection period were included in the study. All patients were admitted to the intensive care unit directly from the emergency department. The diagnosis of OPC poisoning was made on the basis of history and/or evidence of exposure to OPC within the previous 24 hours, characteristic clinical manifestation of organophosphate poisoning (excessive salivation, miosis and fasciculation) and improvement of the signs and symptoms after administration of atropine. All these criteria were required to be fulfilled to be included in the study. The following patients were excluded from the study: 1 . Age < 14 years and $>70$ years; 2 . Patients who has consumed other substances in addition to OPC; 3. Known case of coronary artery disease, hypertension, chronic kidney disease and rheumatic heart disease; and 4. Chronic consumer of alcohol.

The patients were assessed by blood pressure and pulse rate monitoring, electrocardiogram and other relevant investigations as required. They were monitored daily for ECG changes, blood pressure and pulse variations throughout their hospital stay. The severity of poisoning was assessed as per peradeniya organophosphorus poisoning (POP) scale and classified into mild, moderate and severe poisoning. The scale included assessment of pupil size, fasciculation, respiratory rate, pulse rate, seizure and level of consciousness.

After assessment and confirmation of diagnosis, the patients were atropinized by administration atropine intravenously (IV) until achievement of full atropinization which was evaluated based on dry secretions, dilated pupils and tachycardia. The maintenance dose of atropine was given for 2 - 3 days until the patient was stabilized. High dose pralidoxime (PAM) infusion was used as per WHO guidelines. ${ }^{12}$ Pre-structured proforma was used to record the patient's socio demographic and clinical profiles. The age, sex, cause of ingestion, compounds involved, duration of hospital stay, cardiac manifestations and ECG changes at the time of presentation, and during the in-hospital stay were recorded. Cardiac parameters including hypertension (considered as over 140/90 $\mathrm{mmHg}$ ), hypotension (< 90/60 $\mathrm{mmHg}$ ), 
tachycardia ( $>100 \mathrm{bpm}$ ) and bradycardia $(<60 \mathrm{bpm})$ were recorded. ECG was recorded on admission and every 24 hours after that throughout the hospital stay. ECG analysis included rate, rhythm, axis, ST - T changes, PR and QTc interval calculation and conduction abnormalities. QTc was measured as per Bezzet'sfomula and QTc $>0.46$ seconds in male and $>0.47$ seconds in female were considered as prolonged.

\section{Statistical Analysis}

The data was collected and processed in accordance with institutional guidelines to ensure patient privacy and confidentiality. The collected data were entered in Microsoft Excel (2007) and analysed using Statistical Package for Social Science (SPSS) software version 20 by using descriptive statistics (frequency and percentage).

\section{RESULTS}

A total of 54 consecutive patients with OPC poisoning presented to emergency department of NMCTH over a period of 12 months from November 2016 to October 2017. There were $22(40.7 \%)$ males and $32(59.3 \%)$ females. The mean age was $28 \pm 14.5$ ranging from 14 to 75 years. The predominant age group was less than 20 years $(46.3 \%)$ followed by 21 - 30 years (25.9\%) (Table 1). There was female predominance in age group below 40 years (male : female = 1:2) and male predominance in age group more than 40 years $(\mathrm{M}: \mathrm{F}=7: 2)$.

Poisoning was suicidal in $90 \%$ and accidental in $10 \%$ of the patients. The most common OPC was cholropyrifos + cypemethrin (80 \%) followed by dichlorovos (10\%), methylparathiion (10\%).

Pupil size, respiratory rate, heart rate and level of consciousness at presentation are presented in Table 1. According to POP scale, a total of $15(27.8 \%)$ patients were classified as mild poisoning and 39 (72.2 \%) were having moderate poisoning. None of the patients were having severe poisoning.

Cardiac manifestations and ECG changes observed are presented in Table 2. Twenty five (46.3\%) patients were having sinus arrhythmia, among which $11(20.4 \%)$ patients were having sinus bradycardia and 14 (25.9\%) were having sinus tachycardia.

A total of 21 patients (38.9\%) were having additional abnormal ECG findings at presentation (Table 2). After sinus arrhythmia, prolonged QTc interval $(\mathrm{n}=8 ; 14.8 \%)$ and ST $/ \mathrm{T}$ wave changes $(n=6 ; 11.1 \%)$ were predominant ECG abnormalities.

There was no associated elevation of cardiac enzymes in patients with ST/T wave changes. The other additional ECG abnormalities were PVCs ( $\mathrm{n}=4 ; 7.4 \%$ ), prolonged $\mathrm{PR}$ interval $(n=2 ; 3.7 \%)$ and atrial fibrillation $(n=1 ; 1.9 \%)$. The ECG changes as per severity of poisoning are presented in Table 3.

The average ICU stay was three days ranging from two to six days. The average hospital stay was six days ranging from four to 10 days. There was no in hospital mortality.

\begin{tabular}{|ccc|}
\hline & Patient Characteristics & Number (\%) \\
& $<20$ & $25(46.3 \%)$ \\
Age (years) & $21-30$ & $14(25.9 \%)$ \\
& $31-40$ & $6(11.1 \%)$ \\
& $41-50$ & $4(7.4 \%)$ \\
Gender & $51-60$ & $2(3.7 \%)$ \\
& $>$ & $3(5.6 \%)$ \\
Pupil size & Male & $22(40.7 \%)$ \\
& Female & $32(59.3 \%)$ \\
Respiratory rate & Pinpoint & $5(9.3 \%)$ \\
& $<2 \mathrm{~mm}$ & $39(72.2 \%)$ \\
Heart rate & $\geq 2 \mathrm{~mm}$ & $15(27.8 \%)$ \\
& $<20$ & $17(31.5 \%)$ \\
Fasciculation & $\geq 20$ & $37(68.5 \%)$ \\
& $<60$ & $11(20.4 \%)$ \\
Level of & $\geq 60$ & $43(79.6 \%)$ \\
consciousness & Present & $6(11.1 \%)$ \\
Peradeniya & Absent & $48(88.9 \%)$ \\
organophosphate & No response to verbal command & $27(50 \%)$ \\
poisoning Scale & Mild (0 - 3) & $23(42.6 \%)$ \\
\hline \multicolumn{2}{c}{ Table 1. Demographic and Clinical Presentation } \\
\hline \multicolumn{2}{c}{ Conscious, rationale } \\
\hline
\end{tabular}

\begin{tabular}{|ccc|}
\hline Sl. No. & ECG Findings & Number (\%) \\
& Sinus rhythm & $36(66.7 \%)$ \\
1 & Tachycardia & $14(25.9 \%)$ \\
& Bradycardia & $11(20.4 \%)$ \\
2 & Normal rate & $11(20.4 \%)$ \\
& Prolonged QTc & $8(14.8 \%)$ \\
3 & ST / T Changes & $6(11.1 \%)$ \\
& ST elevation & $4(7.4 \%)$ \\
4 & T wave inversion & $2(3.7 \%)$ \\
5 & Premature ventricular complexes (PVCs) & $4(7.4 \%)$ \\
6 & Prolonged PR interval & $2(3.7 \%)$ \\
\hline \multicolumn{3}{c}{ Table 2. ECG Changes in Patients with OPC Poisoning } \\
\hline
\end{tabular}

\begin{tabular}{|cccc|}
\hline SI. No. & ECG Findings & Mild Poisoning (n) & Moderate Poisoning(n) \\
1. & Atrial fibrillation & 1 & Nil \\
2. & Prolonged QTc & 2 & 6 \\
3. & ST changes & 1 & 3 \\
4. & T inversion & Nil & 2 \\
5. & PVCs & 1 & 3 \\
6. & Prolonged PR interval & Nil & 2 \\
7. & Sinus tachycardia & 8 & 6 \\
8. & Sinus bradycardia & 2 & 9 \\
\hline \multicolumn{4}{c}{ Table 3. ECG Changes According to Severity of Poisoning } \\
\hline
\end{tabular}

\section{DISCUSSION}

The clinical presentation of OPC poisoning ranges from cholinergic syndrome to flaccid paralysis, intractable seizures and various cardiovascular effects. The mechanism of cardiac toxicity is multifactorial. Hypoxemia, acidosis and electrolyte derangements, sympathetic and parasympathetic over activity, direct toxicity on myocardium and conduction system of the heart are common predisposing factors. OPC poisoning itself causes diarrhoea and vomiting which can lead to electrolyte derangements that may cause electrocardiographic changes. ${ }^{11}$ Cardiac toxicity after OPC poisoning has been described in three phases: Phase 1. Brief period of increased sympathetic tone, Phase 2. Prolonged period of parasympathetic activity, Phase 3. QTc prolongation followed by torsades de pointes ventricular tachycardia and then ventricular fibrillation. Hypertension and sinus tachycardia are nicotinic while hypotension and sinus bradycardia are cholinergic manifestations. ${ }^{12}$

In our study, the most common ECG abnormality was sinus arrhythmia seen in $46.3 \%$ (sinus tachycardia - $25.9 \%$ and bradycardia - $20.4 \%$ ). Similar findings were observed in the study by Karki et al. ${ }^{10}$ Sinus tachycardia could be related to nicotinic effects of OPC while sinus bradycardia can be 
attributed to muscarinic effects. Usually bradycardia dominates in the early cholinergic phase of the OP poisoning, however sinus tachycardia was a more frequent finding in our study. Some consider hypertension and tachycardia to be indicators of severe poisoning. ${ }^{13}$

After sinus arrhythmia, prolonged QTc interval was the most frequent ECG abnormality observed in $14.8 \%$ of patients. This may induce VT and can be fatal if not identified and intervened on time. This cardiac effect was also the most common finding in several other studies. The frequency of QTc prolongation in these series of OPC poisoning ranged from 20 to $80 \%$ depending on the severity of the poisoning and the type of the toxic agents. ${ }^{10,14,15}$ This complication usually starts during the second to third day and may last up to two weeks post-intoxication. ${ }^{16}$ Polymorphic ventricular tachycardia of the torsades de pointes has been attributable to prolonged QTc interval. Predisposing factors for QT prolongation and development of torsades de pointes include: older ages, female gender, low left ventricular ejection fraction, left ventricular hypertrophy, ischemia and electrolyte abnormalities including hypokalemia and hypomagnesemia. ${ }^{16}$

After sinus arrhythmia and prolonged QTc interval, ST - T wave changes were third most common ECG abnormalities seen in $11.1 \%$ patients. ST-segment elevation was seen in 7.4 $\%$ and $\mathrm{T}$-wave inversion in $3.7 \%$ patients. There was no associated elevation of cardiac enzymes. Very high incidence of ST-T changes ranging from $43 \%$ to $91 \%$ were noted by other investigators.11,17,18 There was associated high mortality and sudden death. This discrepancy is probably due to severe poisoning and high dose of atropine requirement

Ventricular premature complexes (VPCs) was seen in 7.4 $\%$ of patients, however none developed ventricular tachycardia and fibrillation in our study. Administration of atropine in high doses has been implicated in the development of these ventricular arrhythmias. First degree AV block was found in $3.7 \%$ of patients in our study. Various studies have reported AV block ranging from 5.4 to $8.4 \%$ of patients. ${ }^{15,19}$

Non-cardiogenic pulmonary oedema has been reported ranging from $21.6 \%$ to $43 \% .{ }^{13,14}$ However, none developed pulmonary edema and cardiac failure in our study. Studies have shown most of the patients presenting with OP poisoning are in younger age group $(<30$ years $)$ similar to our study where more than two third (72.2\%) patients were in age group $<30$ years. The distribution of gender is variable in different studies however in our study number of female (59\%) were higher than male.

In our study, $27.8 \%$ were having mild score and $72.2 \%$ were having moderate score on paradeniya OPC poisoning score. None were having severe poisoning. All patients were admitted to ICU for monitoring till doses of atropine was stopped and patient was started on oral feeding.

Cardiac complications of OP poisoning are not fully appreciated by many physicians. Mostly they occur during early hours of poisoning for which the patient should be transferred immediately to intensive care unit where proper resuscitative facilities are available. ${ }^{13}$ Intensive care support, meticulous respiratory care, and administration of atropine in adequate doses very early are the keys to manage cardiac toxicity of OPCs.

\section{CONCLUSIONS}

Organophosphorus compounds are the important cause of poisonings in our community. Cardiac complications due to OPC poisoning can be fatal if not treated on time. Identification of ECG abnormalities and timely intervention can prevent cardiac complications and sudden cardiac death. The common ECG abnormities found in our population were sinus arrhythmia, prolonged QTc, ST elevation, T wave inversion, PVCs and prolonged PR interval and atrial fibrillation.

Data sharing statement provided by the authors is available with the full text of this article at jemds.com.

Financial or other competing interests: None.

Disclosure forms provided by the authors are available with the full text of this article at jemds.com.

\section{REFERENCES}

[1] Jeyaratnam J. Acute pesticide poisoning: a major global health problem. World Health Stat Q 1990;43(3):139-44.

[2] International Programme on Chemical Safety, World Health Organization (WHO). Epidemiology of pesticide poisoning: harmonized collection of data on human pesticide exposure in selected countries. Geneva, Switzerland: WHO Press 2004.

[3] Gupta SK, Joshi MP. Pesticide poisoning cases attending five major hospitals of Nepal. J Nep Med Assoc 2002;41(144):447-56.

[4] Paudyal BP. Poisoning: pattern and profile of admitted cases in a hospital in central Nepal. J Nepal Med Assoc 2005;44(159):92-6.

[5] Eddleston M, Karalliedde L, Buckley N, et al. Pesticide poisoning in the developing world - a minimum pesticides list. Lancet 2002;360(9340):1163-7.

[6] Singh S, Sharma N. Neurological syndromes following organophosphate poisoning. Neurol India 2000;48(4):308-13.

[7] Noshad H, Ansarin K, Ardalan MR, et al. Respiratory failure in organophosphate insecticide poisoning. Saudi Med J 2007;28(3):405-7.

[8] Wadia RS, Sadagopan C, Amin RB, et al. Neurological manifestations of organophosphorous insecticide poisoning. J Neurol Neurosurg Psychiatry 1974;37(7):841-7.

[9] Anand S, Singh S, Saikia UN, et al. Cardiac abnormalities in acute organophosphate poisoning. ClinToxicol (Phila) 2009;47(3):230-5.

[10] Karki P, Ansari JA, Bhandary S, et al. Cardiac and electrocardiographical manifestations of acute organophosphate poisoning. Singapore Med J 2004;45(8):385-9.

[11] Saadeh AM, Farsakh NA, Al-Ali MK. Cardiac manifestations of acute carbamate and organophosphate poisoning. Heart 1997;77(5):461-4.

[12] Ludomirsky A, Klein HO, Sarelli P, et al. Q-T prolongation and polymorphous ("torsade de pointes") ventricular arrhythmias associated with organophosphorus insecticide poisoning. Am J Cardiol 1982;49(7):1654-8. 
[13] Megarbane B. Toxidrome-based approach to common poisonings. Asia Pac J Med Toxicol 2014;3(1):2-12.

[14] Yurumez Y, Yavuz Y, Saglam $H$, et al. Electrocardiographic findings of acute organophosphate poisoning. J Emerg Med 2009;36(1):39-42.

[15] Paul UK, Bhattacharyya AK. ECG manifestations in acute organophosphorus poisoning. J Indian Med Assoc 2012;110(2):98, 107-8.

[16] Bar-Meir E, Schein 0, Eisenkraft A, et al. Guidelines for treating cardiac manifestations of organophosphates poisoning with special emphasis on long QT and
Torsades De Pointes. Crit Rev Toxicol 2007;37(3):27985.

[17] Dalvi CP, Abraham P, Iyer SS. Correlation of electrocardiographic changes with prognosis in organophosphorus poisoning. J Postgrad Med 1986;32(3):115-9.

[18] Mukherjee M. Cardiotoxicity profile in organophosphate and carbamate poisoning. Indian Heart Journal 1999;51:662-9.

[19] Kiss Z, Fazekas T. Arrhythmias in organophosphate poisonings. Acta Cardiol 1979;34(5):323-30. 